\title{
The influence of distance to shops on the quality of diet of women of childbearing age
}

\author{
C. Black ${ }^{1}$, M. Barker ${ }^{1}$, H. Inskip ${ }^{1}$, M. Jarman ${ }^{1}$, W. Lawrence ${ }^{1}$, C. Cooper ${ }^{1}$, G. Moon ${ }^{2}$, J. Baird ${ }^{1}$ \\ and The Food Choice Group \\ ${ }^{1}$ MRC Epidemiology Resource Centre, University of Southampton, Southampton SO16 6YD, UK and ${ }^{2}$ School of Geography, \\ University of Southampton, Southampton SO17 1BJ, UK
}

Many women of childbearing age in the UK have poor-quality diets, and this has important implications for the health and development of their offspring. Recent evidence has suggested that socio-economic and individual level factors including educational attainment, and sense of control are important predictors of the dietary quality in women of this age $\mathrm{e}^{(1,2)}$. However, we have only limited knowledge regarding the influence of environmental factors on the diets of women. Recent research from the USA has suggested that proximity to and the density of food stores are environmental features that influence dietary quality ${ }^{(3)}$. However, there has been little work to examine the relationship between the distance to food shops and the diets of individuals in the UK.

We examined the relationship between the distance from women's homes to the shop or supermarket that they used for their main weekly shop, and the quality of their diets. We carried out a cross-sectional survey of 921 women, median age 30 years, assessing their quality of diet using a validated FFQ, the location of the shop they used for their main weekly shop, and a range of socio-demographic and individual factors. The women lived in three geographical areas within Hampshire: Southampton City, Gosport and Havant. Data from the FFQ were used to produce a standardised score which reflects dietary quality using methods developed as part of the Southampton Women's Survey ${ }^{(4)}$. Postcode information derived from these data was then used to map residential and main shop addresses. ARC-GIS was used to derive shortest-route road distances between the residence and main shop.

Over half of the women lived less than $4 \mathrm{~km}$ from the shop where they did their main weekly shop, with the majority (95\%) living less than $10 \mathrm{~km}$ away. In univariate analyses, the distance to shops was a significant predictor of women's dietary quality, such that women who lived closer to their main shop tended to have diets of poorer quality: for every extra kilometre travelled, the prudent diet score increased by $0.037 \mathrm{SD}, 95 \% \mathrm{CI} 0.001$ to $0.07, P=0.046$. After adjustment for individual and socio-economic factors, the relationship strengthened such that diet score increased by 0.044 sD per km travelled, $95 \%$ CI 0.009 to $0.079, P=0.015$.

These findings suggest that the food environment has a significant influence on the quality of women's diets. Specifically, living closer to the shops where women buy most of their food seems to adversely affect the quality of their diets. One interpretation of these findings is that the food shops in close proximity to residential areas do not provide the kinds of foods that would make it easy to maintain a healthy diet. This interpretation is supported by work from the US which has demonstrated limited availability of healthy food in small urban food stores ${ }^{(3)}$. Interventions to improve the diets of women of childbearing age need to address factors in the food environment as well as individual vulnerabilities.

1. Robinson SM, Crozier SR, Borland SE et al. (2004) Impact of educational attainment on the quality of young women's diets. Eur J Clin Nutr 58, $1147-1180$.

2. Barker M, Lawrence W, Crozier S et al. (2009) Educational attainment, perceived control and the quality of women's diets. Appetite 52, 631-636.

3. Gittelsohn J \& Sharma S (2009) Physical, consumer, and social aspects of measuring the food environment among diverse low-income populations Am J Prev Med 36, 4 Suppl., S161-S165.

4. Crozier SR, Inskip H, Barker M et al. (2010) Development of a shortened FFQ to assess a 'prudent' dietary pattern amongst women in Southampton. Eur J Clin Nutr 64, 99-104.

5. Laska MN, Borradaile KE, Tester J et al. (2009) Healthy food availability in small urban food stores: a comparison of four US cities. Public Health Nutr 13, 1031-1035. 